\title{
Impact of improved client-provider interaction on women's achievement of their fertility goals [Arabic]
}

Frontiers in Reproductive Health

Follow this and additional works at: https://knowledgecommons.popcouncil.org/departments_sbsr-rh How does access to this work benefit you? Let us know!

\section{Recommended Citation}

"Impact of improved client-provider interaction on women's achievement of their fertility goals [Arabic]," FRONTIERS Summary of the intervention, Phase 1. Cairo: Population Council, 2002. 


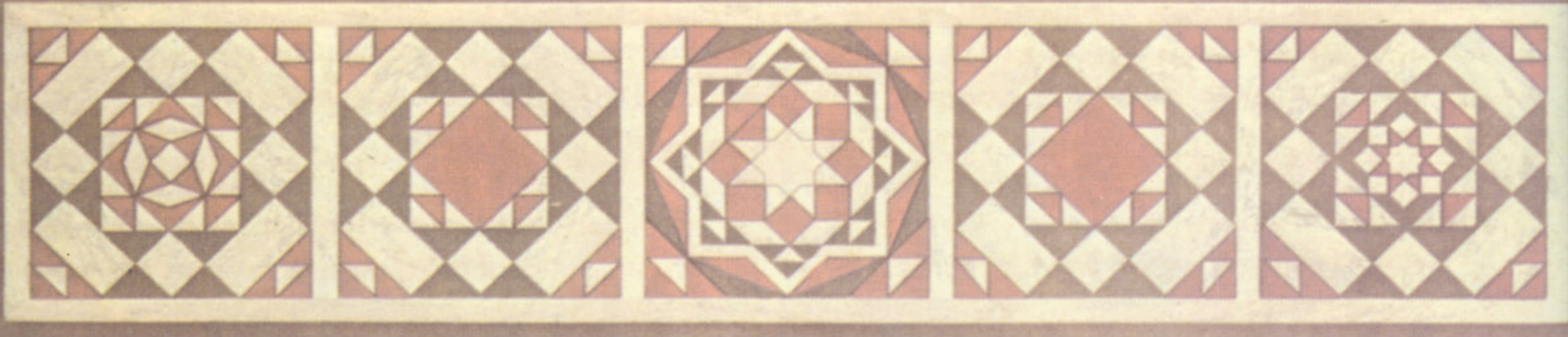

\section{Population Council 7 Frontiers}

$$
\begin{aligned}
& \text { أثر تحسين جودة التعامل والتفاعل }
\end{aligned}
$$

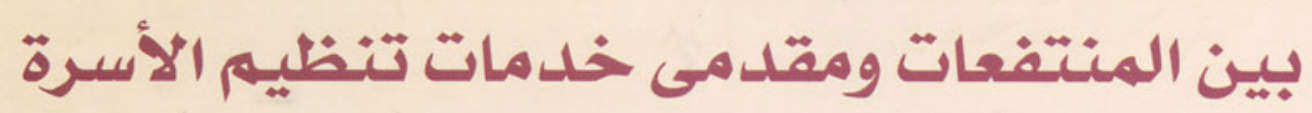

$$
\begin{aligned}
& \text { على تحقيق السيدات لأهدافهن الإنجابية }
\end{aligned}
$$

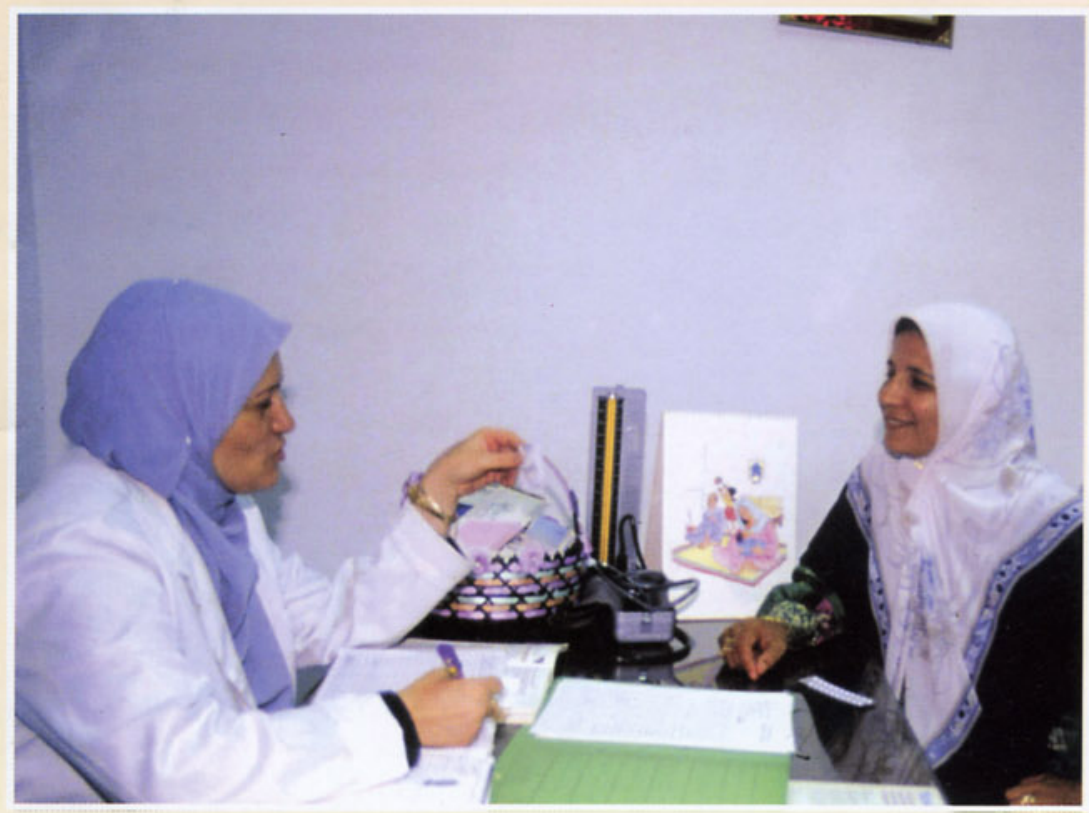

$$
\text { ملخص لتدخل الدراسـة }
$$$$
\text { اكتوبر r. }
$$
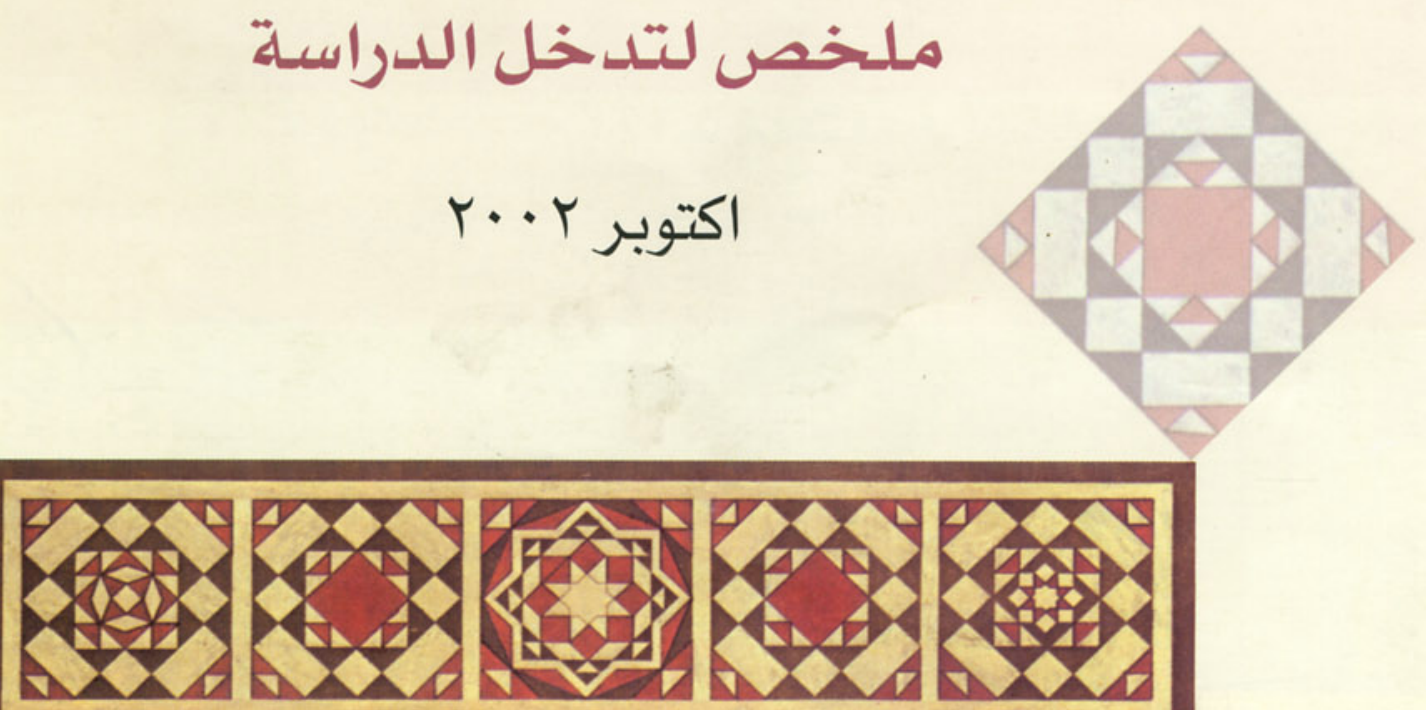


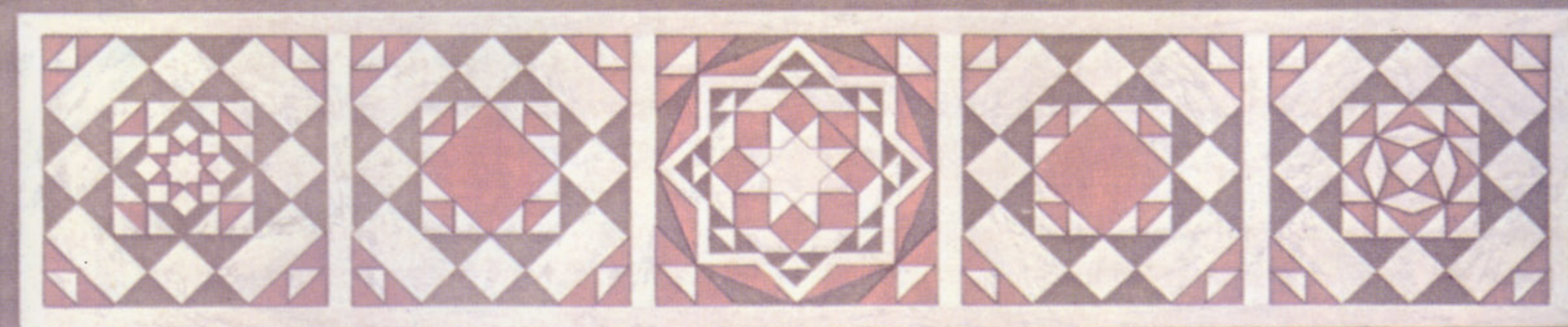

خلفية الدراسة

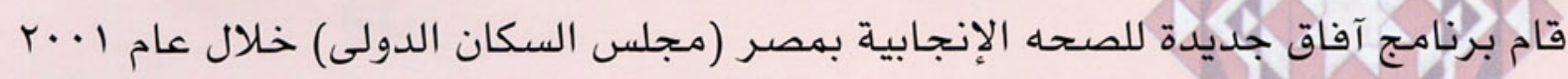

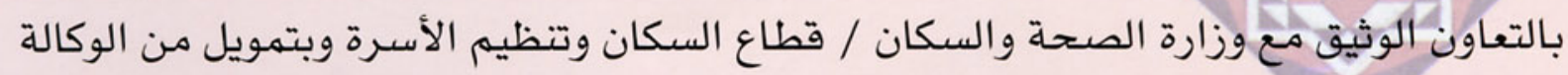

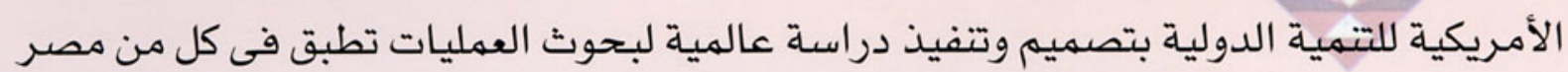

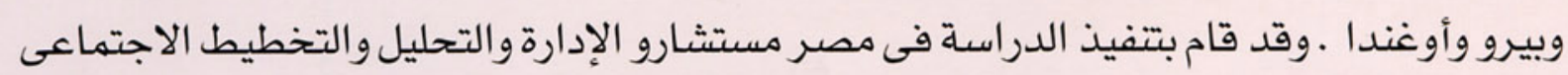

$$
\text { هبدف الدراسـة ) }
$$

تحسين جودة التعامل والتفاعل بين مقدمى الخدمة والمنتفعات بهدف الارتقاء بخدمات تتظيم الأسرة ورضاء المنتفعات عن الخدمات المقدمة والوسائل المستخدمة مما سيكون لهاته تأثيراً إيجابياً

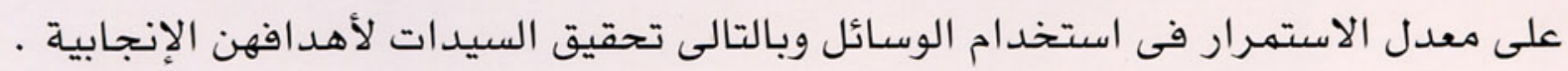

أنشطة الدراسـة

لتحقيق أهداف الدراسة تم تصميم وتتفيذ تدخلات الدراسة على ثلاث مستويات ومن خلال

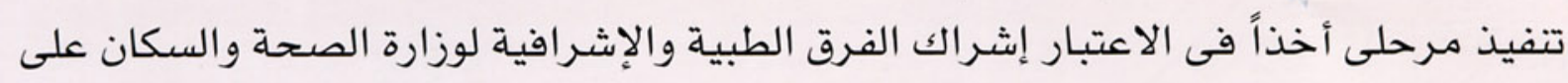

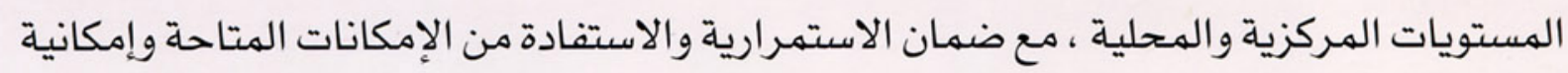

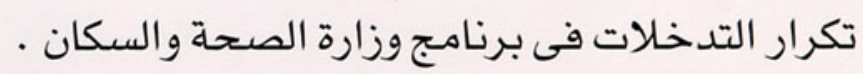
تضمنت مستويات التدخلات ما يلى : أولاً : تدخلات خاصة بالنظم وذلك عن طريق • استثارة اهتمام مديرى ومشرفى تتظيم الأسرة على جميع المستويات حول أسباب وأبعاد مشكلة التعامل والتفاعل وانعكاسها على مستوى تقديم الخدمات للمنتفعات • استحداث واستخدام قائمة إشرافية تتضمن بنود متعددة للاستخدام بواسطة الفرق الإشرافية

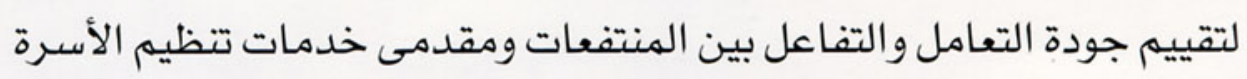
• تدريب مديرى ومشرفى تتظيم الأسرة على التقنيات الحديثة فى تتظيم الأسرة واللازمة

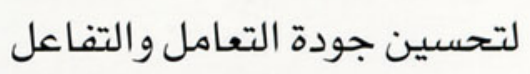

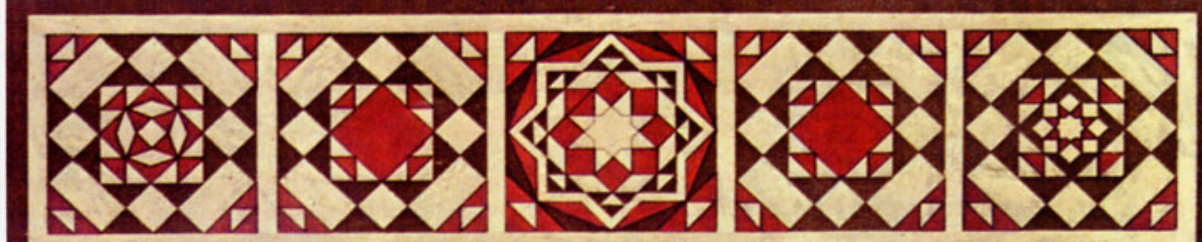




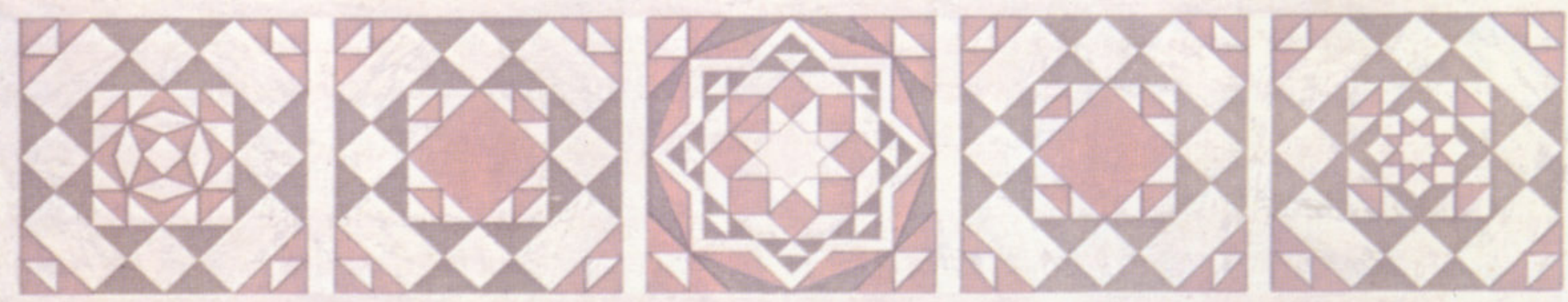

• تقعيل دور الإشراف وذلك بتطبيق الإشراف التيسيرى Facilitative Supervision) • استحداث نظام غير مادى لتحفيز مقدمى الخدمة استتاداً على الإنجازات المحققة فى تحسين جودة التعامل والتفاعل ثانياً : تدخلات خاصة بمقدمى الخدمة وذلك عن طريق • التدريب المستمر للارتقاء بمستوى جودة التعامل والتفاعل وذلك بتطبيق التدريب الشامل فى موقع العمل (Whole-site Training) • تحديث التقنية العلمية لمقدمى خدمات تتظيم الأسرة عن وسـائل تتظيم الأسـرة والأداء الإكلينيكى • تدريب مقدمى الخدمة على تطبيق التقييم الذاتى ووضع الأهداف

(Self-assessment and Goal Setting) • إمداد مقدمى الخدمة بأدوات تعينهم فى تحسين الأداء مثل كتيبات المشورة لاستخدام كل من الأطباء والممرضات

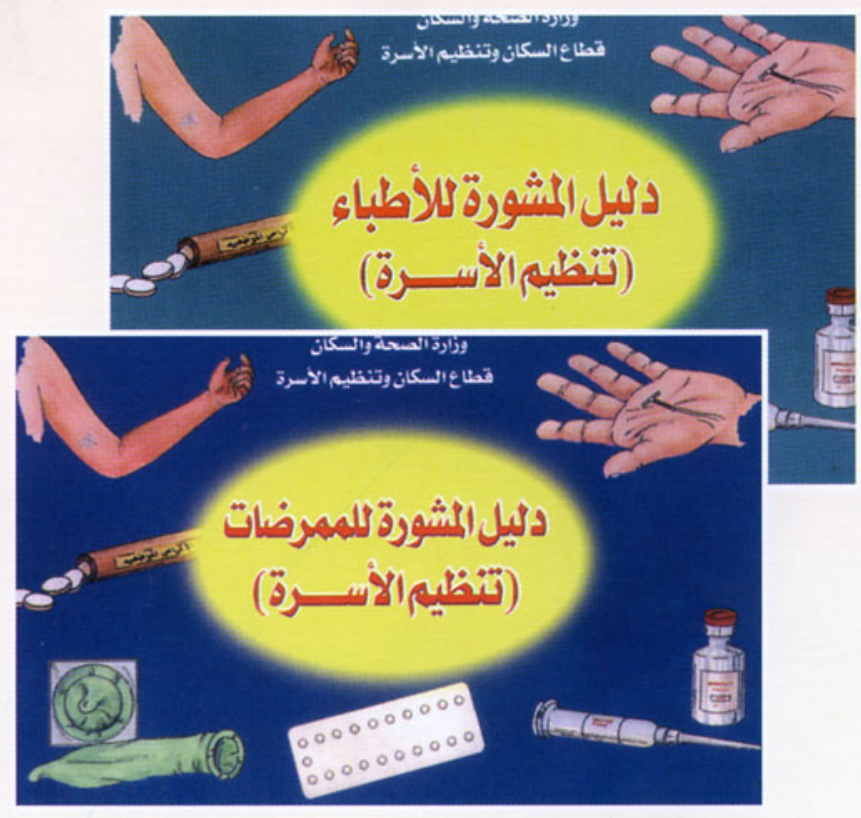
ثالثاً : تدخلات خاصة بالمنتفعات وذلك عن طريق • حث وتمكين المنتفعات من المشاركة الإيجابية وتوجيه أسئلة إلى مقدمى الخدمة للحصول على الهـ أية معلومات يحتجن إليها وذلك عن طريق ملصقات تعرف المنتفعة بحقوقها ( الحصول على خدمة جيدة / الحصول على الوسيلة التى

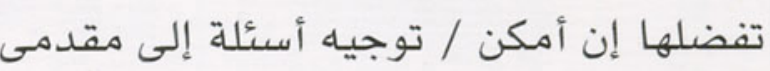
الخدمة والحصول على إجابات لها ) إنهابه إنها • تفعيل دور مقدمى المشورة فى هذا الخصوص S 


\section{مواقع تنفيـ الدراسـة}

تم إجراء الدراسـة فى أربع محافظات فى الوجه البحرى : الشرقية والهنوفية ( تجريبية ) - كفر الشيخ والبحيرة ( ضابطة ) ثم تم اختيار إدارتين من كل محافظة ثم ست مراكز لتتظيم الأسرة من كل إدارة تمثل جميع مستويات تقديم خدمة تتظيم الأسرة فى وزارة الصحة والسكان .

\section{مراحل تنفيذ تدخالات الدراسـة}

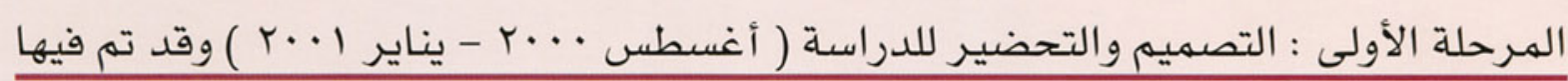

1- تصميم وتنفيذ الدراسة التشخيصية (Diagnostic Study) الهدف من هذه الدراسـة هو التعرف على الوضع الحالى لمستوى التعامل والتفاعل بين مقدمى الخدمة والمنتفعات، وكذلك آراء مقدمى الخدمة والمستوى الإشرافى عن كيفية الارتقاء بهذه الأوضاع وقد تم فى هذه المرحلة إجراء مناقشات بؤرية مع المنتفعات، ومقابلات تفصيلية مع مديرى مشرفى - مقدمى خدمات تتظيم الأسرة وماحلاحظة عدد من المقابلات بين المنتفعات ومقدمى الخدمة ـ وقد تم تحليل هذه المعلومات ومناقشتها في مقابلات لعصف الذهن مع المسئولين على مستوى الهحافظات التجريبية وذلك بهدف التوصل إلى أسباب انخفاض مستوى التعامل والتفاعل مع المنتفعات ومحاولة الوصول إلى اقتراحات لتحقيق مستويات أفضل .
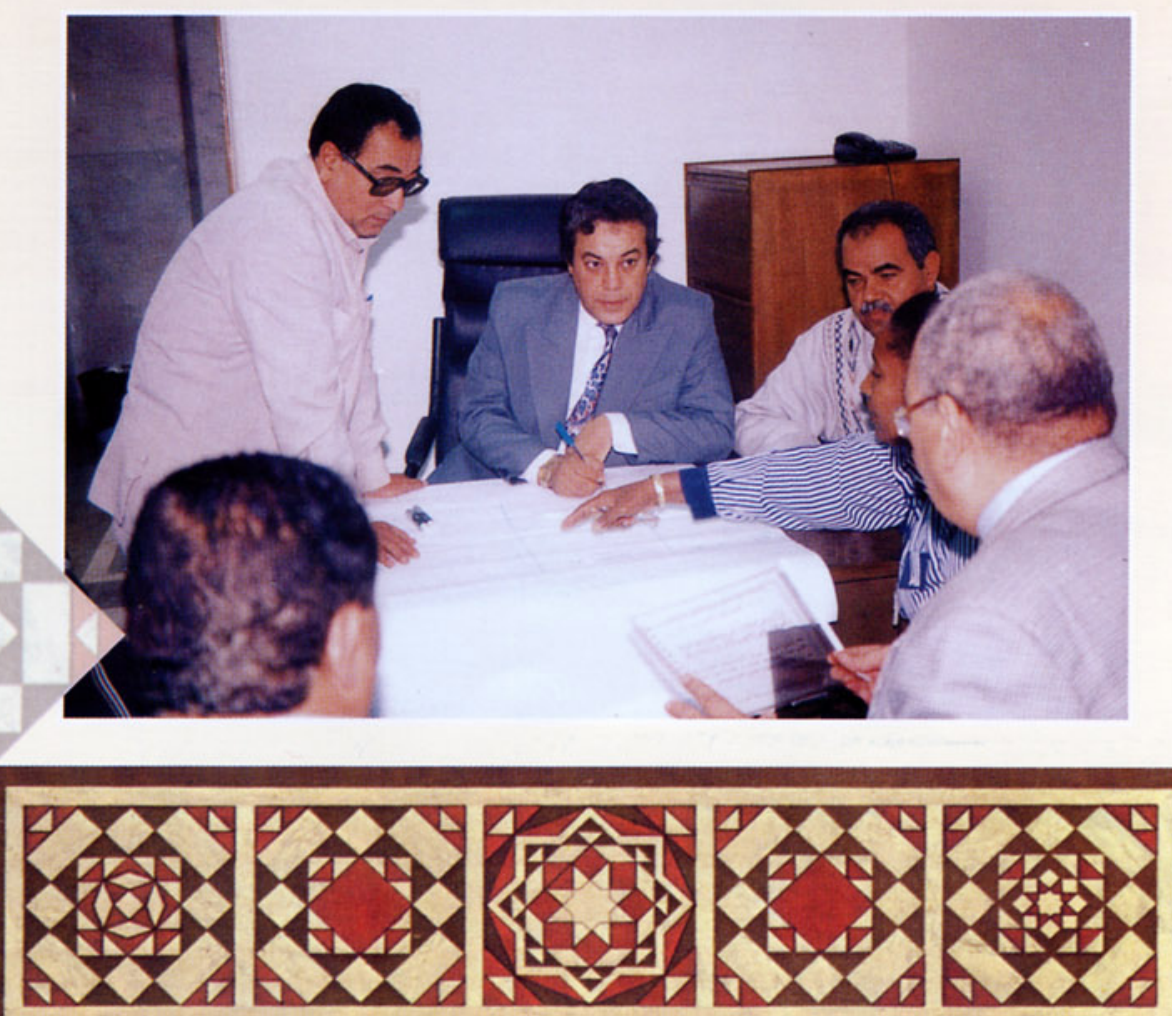


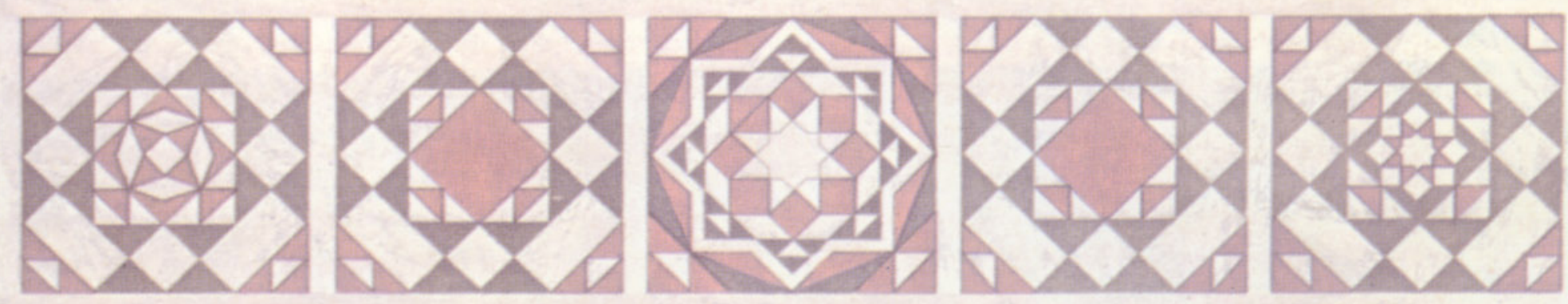

r- التخطيط للتدخلات

تم ذلك من خلال مراجعة نتائج الدراسـة التشخيصية والحلول المقترحة ومراجعة المراجع العلمية والدراسـات السابقة المحلية والعالمية وكذا مراجعة بروتوكولات تقديم الخدمة الخاصة بوزارة الصحة والسكان

r- استحداث قائمة الإشـراف الخاصدة بجودة التعامل والتفاعل وكذا نماذج المتابعة والإشراف صممت قائمة الإشراف الأسـاسية بالاستعانة بالمراجع السابق ذكرها وبعد مراجعة استمارة

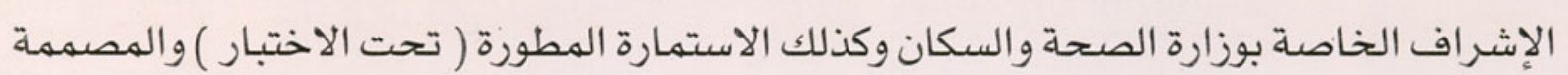
بواسطة الباثفايندر (Pathfinder International / POP IV project) وقد شملت هذه القائمة VV معياراً مقسمـة إلى سبعة أجزاء كل جزء يحتوى على عدد من المعايير الخاصة . ولقد استحدثت هذه القائمـة للاستعانة بها في تحليل الوضع (Situation Analysis) في إنى العيادات وتحديد جوانب القصور قبل البدء فى الدراسـة وكذلك متابعة الأداء شهرياً حتى نهاية الدراسـة بواسطة مقدمى الخدمة وبمساعدة المشرفين ·.وقد شملت أدوات الإدارة أيضاً نماذج للتعرف على احتياجات عيادات تتظيم الأسـرة وكذلك أنشطة التخطيط والمتابعة على مستوى كل عيادة ، بالإضافة إلى التوجيهات التى يسجلها فريق الإشراف خـلال

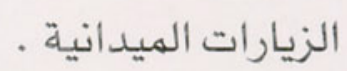

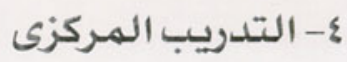

تم تدريب عدد ^/ من مديرى ومشرفى تتظيم الأسـرة على مستوى المحافظات والإدارات المشاركة فى الدراسـة التجريبية لمدة أسبوع فى المركز الإقليهى للتدريب على الصحة الإنجابية . وكان الهدف من هذا التدريب هو إيجاد كادر من المديرين والمشرفين المتميزين فى جودة الأداء فى التعامل والتفاعل قادرين على نقل هذه المهارات لمقدمى الخدمة من خالال عملهم وذلك لكونهم حجر الأسـاس فى تتفيذ الدراسة فى أماكن البحث .
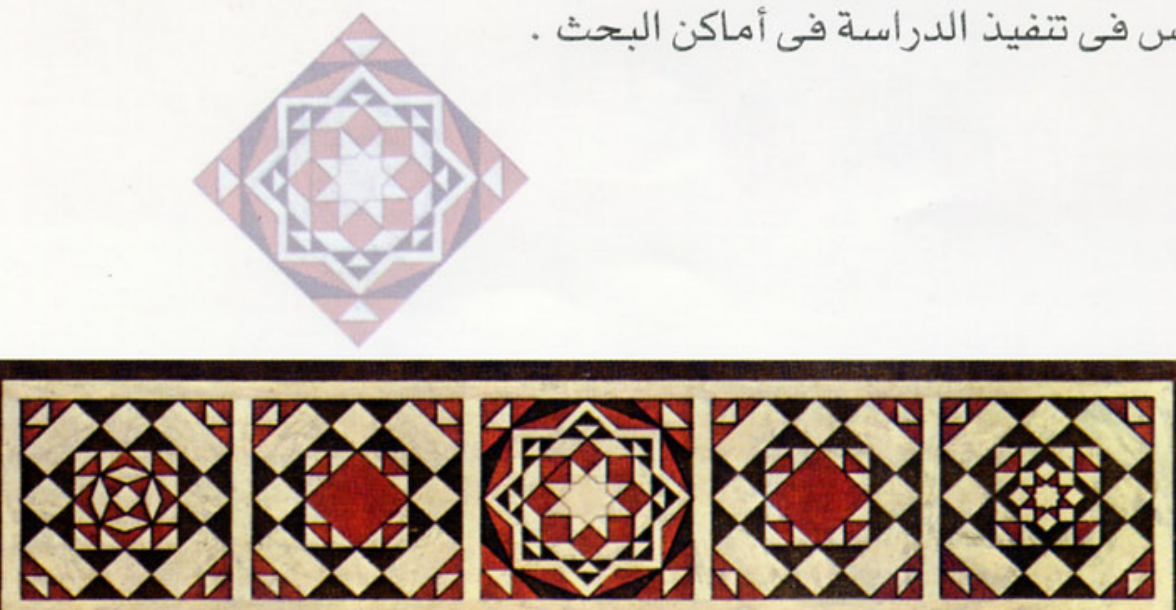


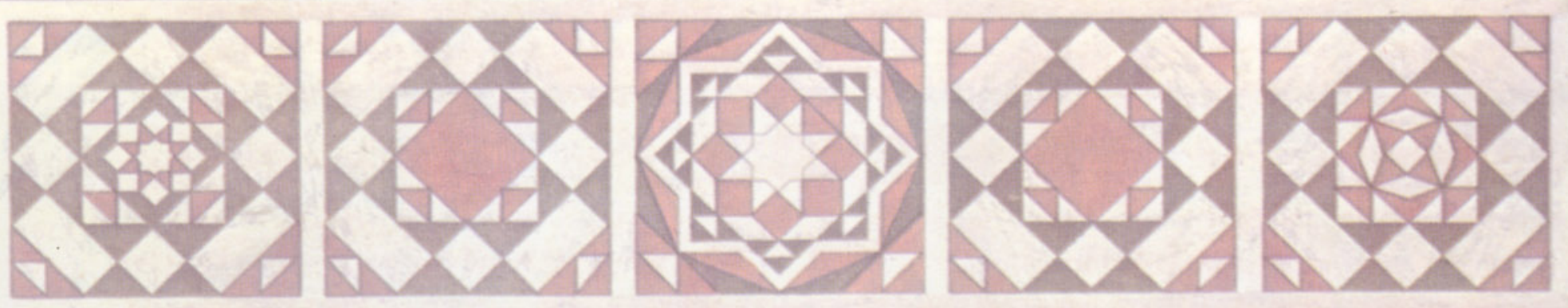

وقد روعى فى التدريب استخدام الأساليب التفاعلية وتمثيل الأدوار وحلقات النقاش وعصف الذهن. وتم دمهج تدريب الأطباء والممرضات فى كل المحاضرات النظرية والعملية وذلك للتوحيد ولخلق الشعور العام بأن إيجاد روح الفريق لابد أن يبدأ من الفريق الإشرافى . ومن أجل قياس التغيير الناتج عن التدريب فى المعلومات والههارات المكتسبة أجرى للمتدربين تقييم قبلى وبعدى ( قبل وبعد التدريب) وكذلك قبل وبعد كل جلسة تدريبيه .

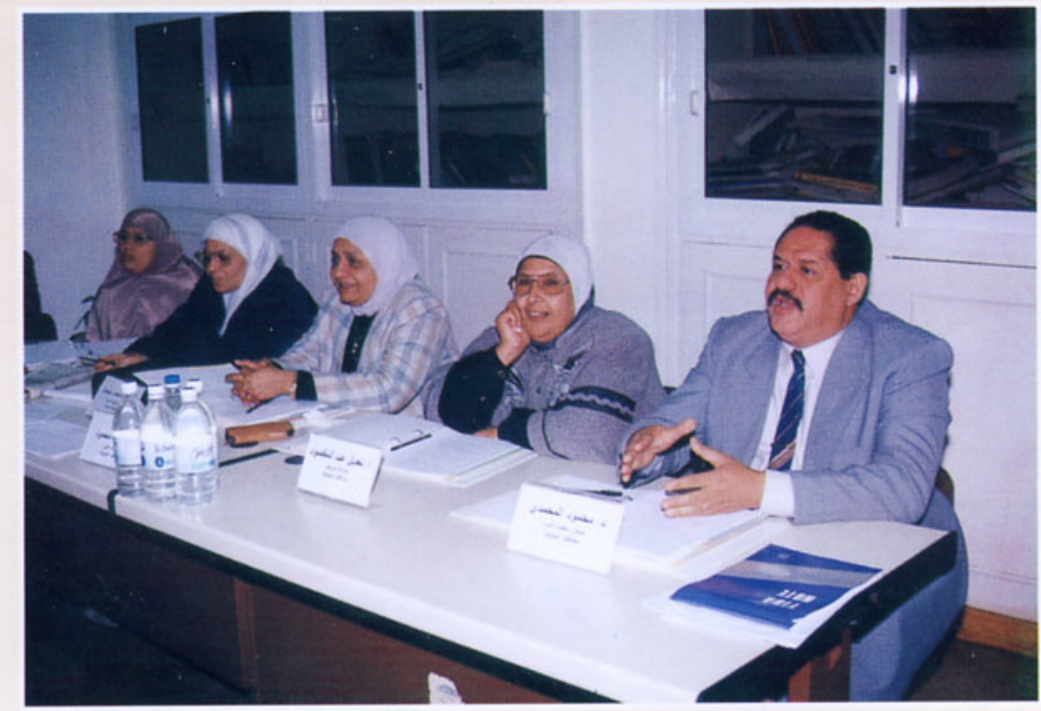

وقد اشتمل التدريب على الموضوعات الآتية : • يفية تحسين الاتصال بين مقدمى الخدمة والمنتفعات فى عيادات تتظيم الأسـرة • تحديث المعلومات حول وسـائل تتظيم الأسرة • الاختيار القائم على الهعرفة

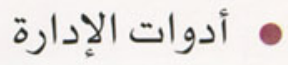
• استعمال وسـائل التقييم الذاتى وأسلوب وضع الأهداف

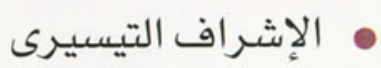
• التدريب الشـامل فى موقع العمل وتحديد الاحتياجات التدريبية

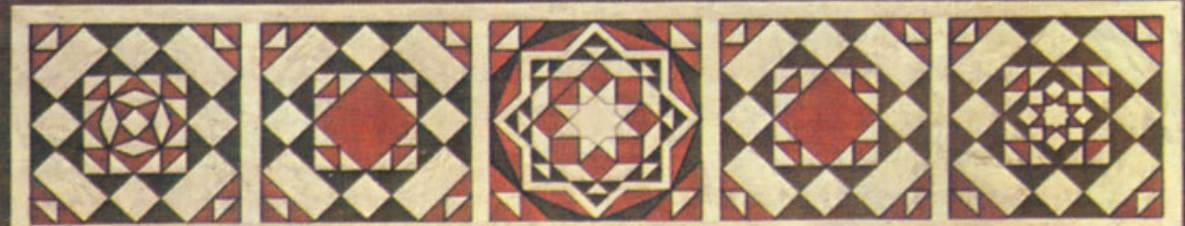




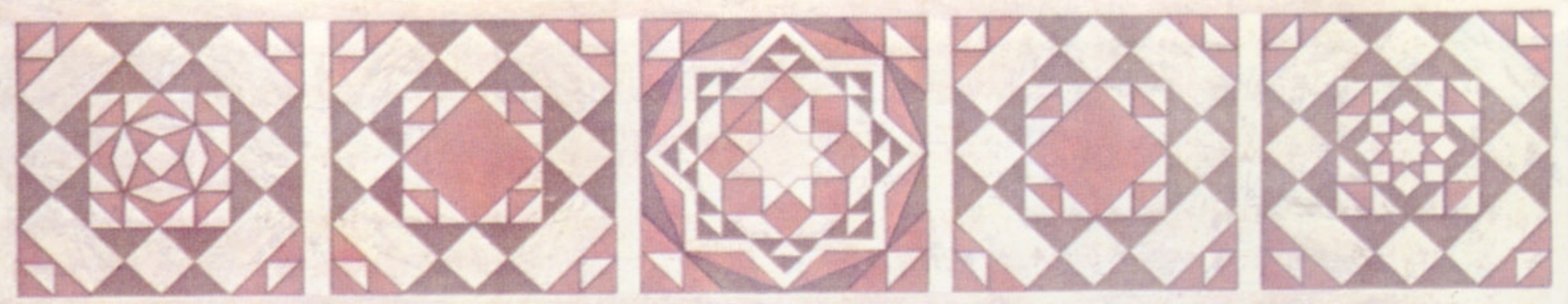

ه- إعداد معينات التعامل والتفاعل متسقة مـع المواد المتوفرة بوزارة الصحة والسكان عن الإعلام والتعليه والاتصال

وقد تم إعداد ملصقات تهدف إلى حث المنتفعات على أن توجه أسئلة إلى مقدمى الخدمة إذا رغبت فى ذلك وتعرفها بحقوقها وخاصة الحصول على خدمة جيدة وكذلك تم إعداد دليل للمشورة خاص بالطبيب وبالممرضة وتم ذلك بعد مراجعة وموافقة وزارة الصحة والسكان على المحتوى.

T- استحداث وسيلة غير مادية للتحفيز

اتفق على أن تكون المفاضلة بين العيادات المشاركة على مستوى الإدارات وأن يؤخذ فى الاعتبار الأداء المتميز بالنسبة للعيادة وينال مقدمو الخدمة فى العيادة المختارة وكذلك فريق الإشراف

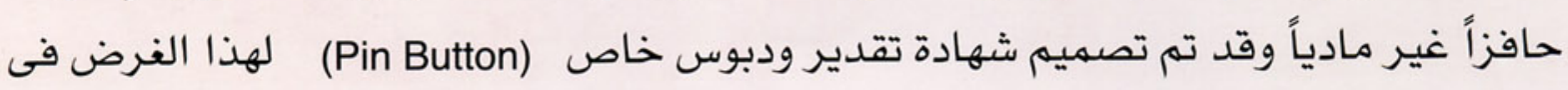
الدراسـة .

المرحلة الثانية : تتفيذ الدراسة التدخلية ( يناير - أبريل ا +.r ) وقد تم فيها تتفيذ التدخلات

الآتيه

ا- التوجيه / التدريب لمقدمى الخدمـة

قام بذلك الفريق الذى تم تدريبه من المديرين والمشرفين حيث زار كل فريق إشرافى ( مشرف طبى - مشرف تتظيم أسرة - مشرفة تمريض ) المراكز المشتركة فى الدراسة التجريبية وقام كل فريق بشرح الدراسة وأهدافها والدور المتوقع من كل فرد في فريق العمل . وقد تم تتفيذ 1 حلقات تدريبية استمرت كل منها لمدة ثثلاث أيام لعدد 9 • 1 متدرب يمثلون الأطباء - الممرضات - الأخصائيين الاجتماعيين العاملين بالعيادات لتحقيق أهداف رفع كفاءة مقدمى الخدمة من حيث المعلومات والمهارات الخاصة بالتعامل والتفاعل مع المنتفعات وتدريبهم على التى التقييم الذاتى ووضع الأهداف كذلك على كيفية وضع خطط مستقبلية قصيرة المدى وتتفيذها لرفع كفاءة الأداء ـ وقام بالتدريب الفريق الإشرافى باستخدام المنهج التدريبى السابق تتفيذه فى المركز الإقليمى للتدريب على الصحة الإنجابية ولكن بصورة مختصرة .

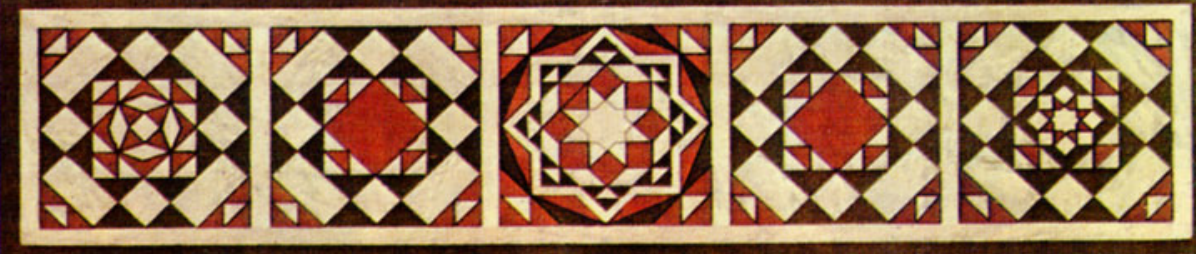




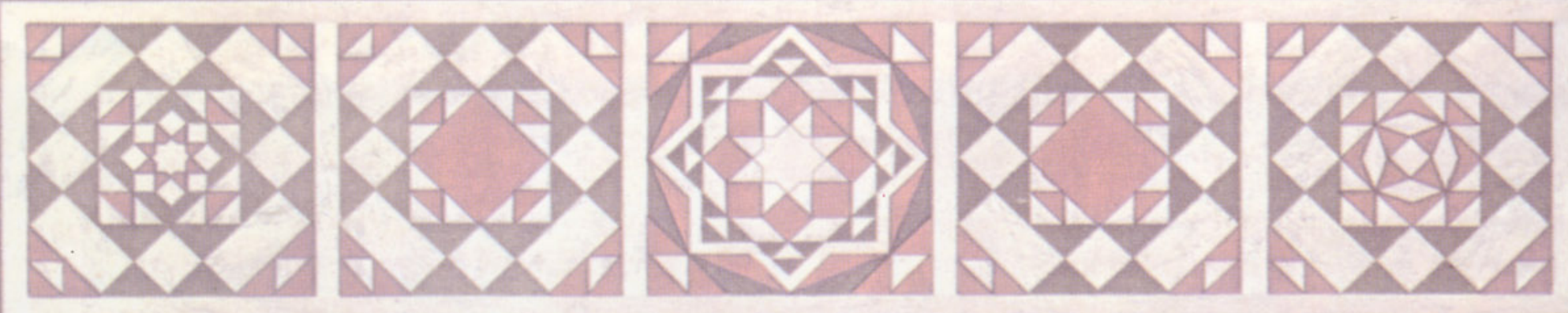

ب- تحديد الاحتياجات

قام مقدمو الخدمة فى العيادات كفريق واحد بمساعدة فريق الإشراف بتحديد الاحتياجات التدريبية وغير التدريبية وتشخيص الموقف ووضع الخطط المستقبلية بحيث تكون سهلة التتفيذ للتغلب على العراقيل التى تؤثر على جودة الأداء وذلك باستخدام النماذج المعدة لهذا الغرض وتشمل استمارة الإشراف، واستمارة تحديد الاحتياجات . ومن أمثلة العراقيل لتحسين جودة الأداء انخفاض مهارات الاتصال لدى بعض مقدمى الخدمة، عدم دراية بعض مقدمى الخدمة بأهمية شرح خطوات تقديم الخدمة أو أهمية الحصول على موافقة المنتفعة قبل إجراء الفحص الطبى اللازم، بالاضافة إلى القصور فى الأجهزة المتاحة للعيادات وخاصة أجهزة الأوتوكلاف أو الغلايات .

ب- الإشراف التيسيرى والتدريب الشامل فى موقع العمل أ ـ الإشراف التيسيرى

وقد تم من خلال زيارات إشرافية مكثفة من قبل المشرفين والمديرين كوسيلة لتفعيل دور الزيارات الإشرافية وذلك عن طريق : المباشرة الدورياة

حل المشكلات مع فريق مقدمى الخدمة بالمـراكز الاتصال المتبادل بين المشرفين ومقدمى الخدمة ( المناقشات التيسيرية - الاتصال الثنائى

$$
\text { ردود الفعل والإنصات الفعال ) }
$$$$
\text { ولقد تم خلال الزيارات الإشرافية : }
$$
• تلبية احتياجات مقدمى الخدمة بشأن الإدارة والإشراف الجيد • تلبية احتياجات مقدمى الخدمة بشأن جودة الإمدادات والبنية الأسـاسية للعيادات • تلبية احتياجات مقدمى الخدمة من المعلومات والتدريب والتطوير • وضع أدوات حل المشكلات فى أيدى العاملين والتحفيز على العمل كفريق

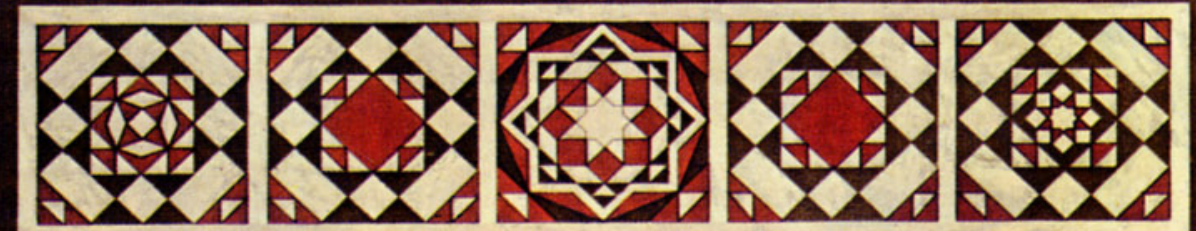


ب. ب التدريب الشامل فى موقع العمل قام بذلك فريق الإشراف من خلال فهم واف لإمكانيات مقدمى الخدمة واحتياجاتهم التدريبية والسياق الذى يعملون بداخله وريطه على نحو وثيق بنظام الإشراف وقد تم الاستجابة للاحتياجات

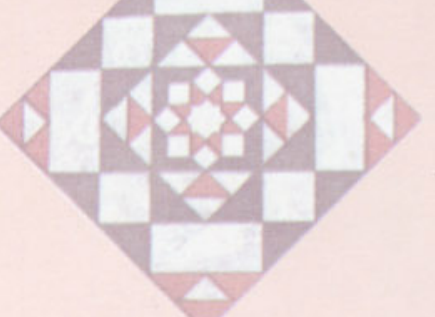

$$
\begin{aligned}
& \text { التدريبية من خلال : } \\
& \text { • التدريب على المهارات } \\
& \text { • تحديث المعلومات } \\
& \text { • التوجيه وجذب الانتباه }
\end{aligned}
$$

ولقد أمكن تدريب وتطوير أداء فريق العمل وأصبح المتدربون مسئولين عن مشاركة معرفتهم

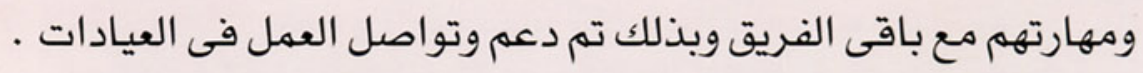

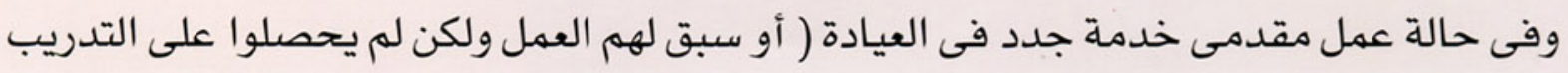

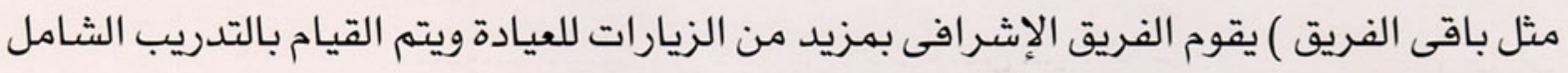

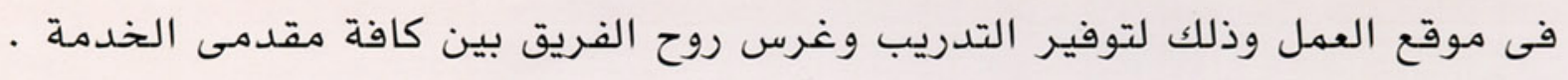

$$
\text { ع- التقييم الذاتى وأسلوب وضع الأهداف }
$$

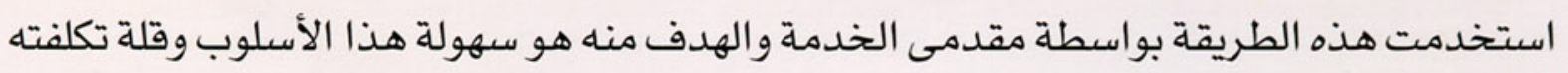

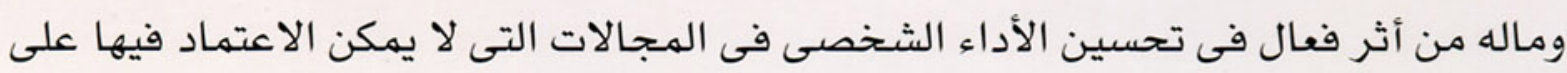

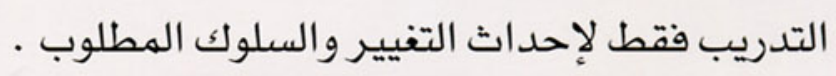

ولهذا الغرض استخدمت استمارة تتكون من خمسة أجزاء يختص كل جزء منها بمهارة من مهارات

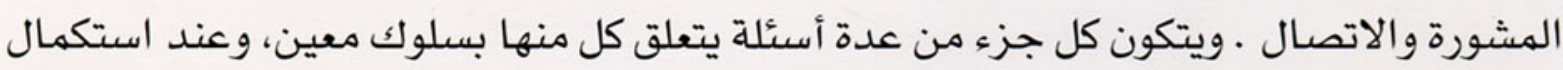

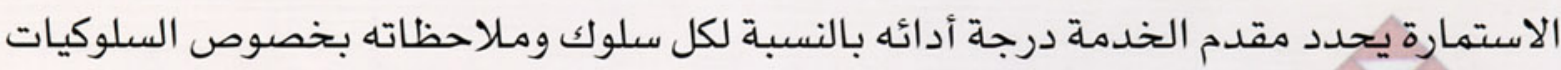

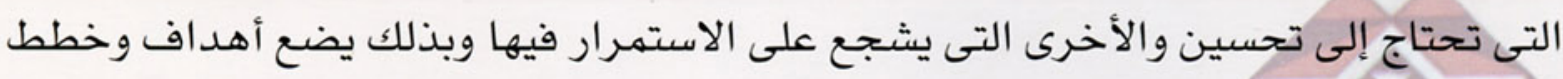

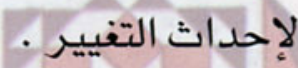
كان على مقدم الخدمة مله هذه الاستمارة أسبوعياً، الأمر الذى لا يستغرق أكثر من · ال دقائق

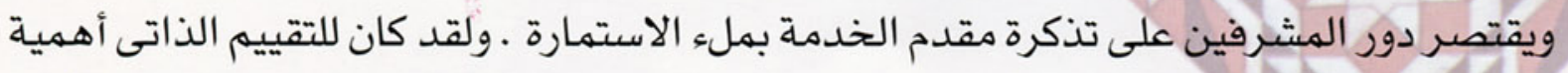

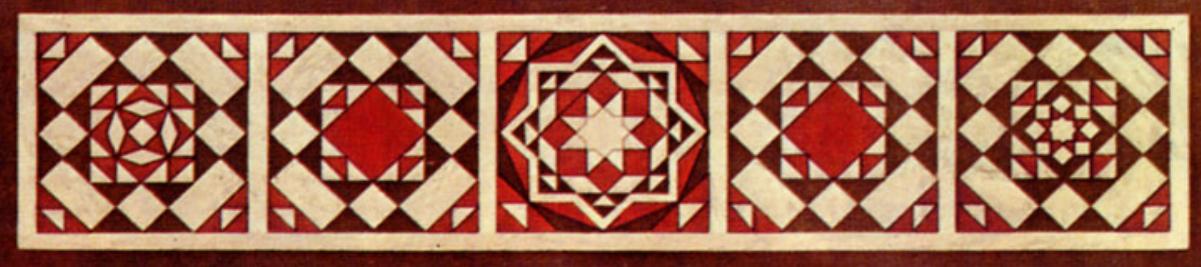




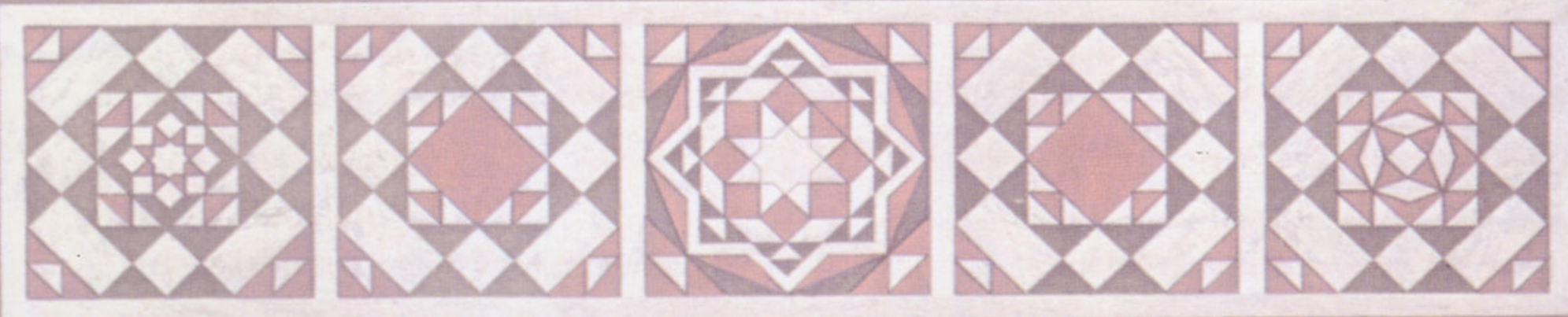

فى مساعدة مقدمى الخدمة فى الارتقاء بمهاراتهم وإعطائهم الشعور بالاستقلالية والرضا والمسئولية تجاه المنتفعات . وكان مقدم الخدمة يحتفظ بهذه الاستمارة بشكل خاص ورلا يطلع عليها فريق الإشراف .

\section{ه- الارتقاء بالبيئة المصاحبة والبنية الأساسية للعيادات}

من أجل الحصول على مخرجات إيجابية للتدخلات قامت الدراسة أيضاً بتنظيم وتوفير الحد الأدنى من البيئة المصاحبة والبنية المـلائمة الواجب توافرها لتوفير عناصر الأداء الأمثل فى العيادات من خلال تشتجيع المبادرات الشخصية والجماعية لفرق العمل بدعم من الفريق الإشرافى أخذاً فى الاعتبار الامكانات المتاحة على مستوى العيادات والإدارات . وكان الهدف الأسـاسى من هذه الأنشطة هو توفير جو من الخصوصية للمنتفعات والراحة وسرية المعلومات المتداولة بينهم وبين مقدمى الخدمة . وقد أقتصر دور فريق البحث على توفير الإمدادات المالية البسيطة لبعض الأنشطة اللازم تتفيذها .

$$
\text { 7- تبادل الخبرات }
$$

وتم ذلك من خلال أربعة لقاءات على مستوى الإدارة بحضور فرق العمل فى العيادات الستة المشاركة فى كل إدارة وحضور فرق الإشراف للإدارتين ومديرى تتظيم الأسرة بالمحافظة وقد تم من خلال هذه اللقاءات ما يلى :

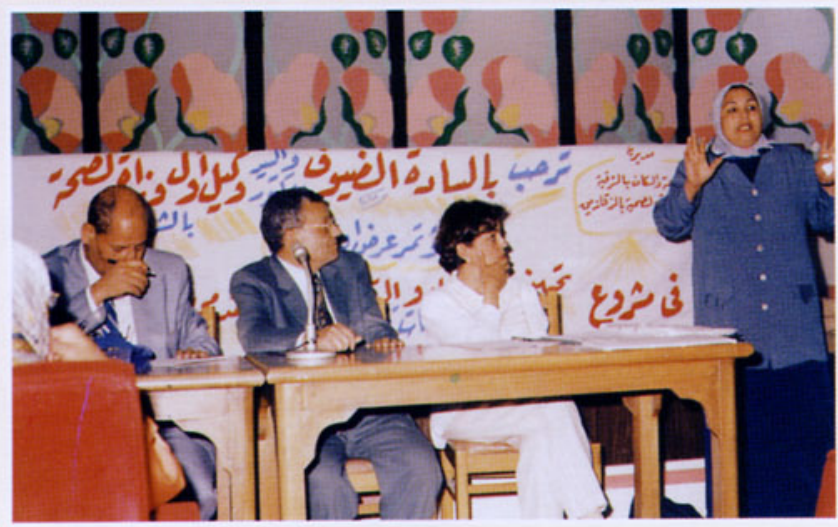

• تبادل الخبرات بين مقدمى الخدمة بالنسبة للعيادات فى الإدارة الواحدة وبين فريقى الإشراف فى الإدارتين حيث تم عرض الإنجازات التى تمت على مستوى الإدارة ثم العيادات وطرق حل المشاكل والهجهودات التى بذلت لتحقيت الأهداف ثم قامت كل عيادة بتحديد الخطط والأهداف التى يجب تحقيقها للارتقاء الأمثل بالأداء.

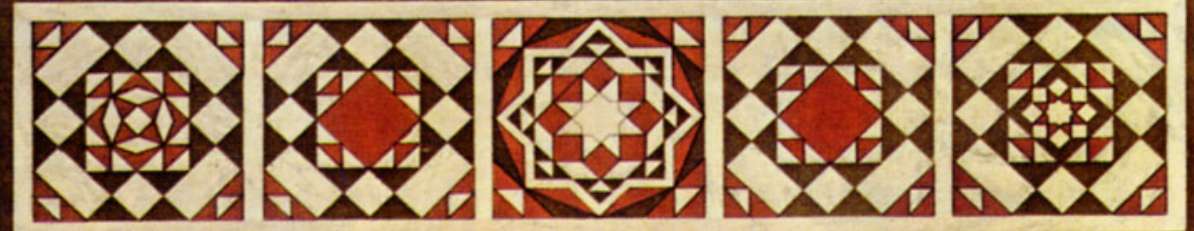




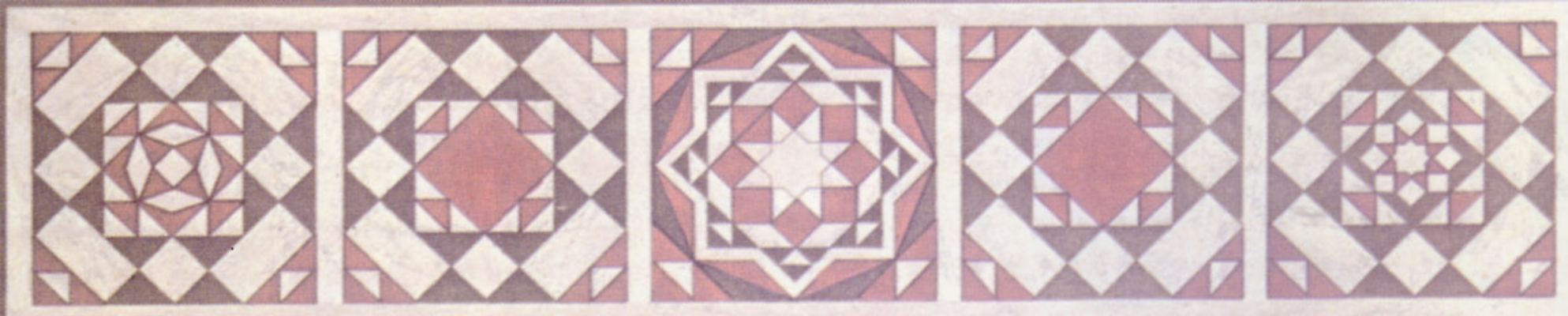

• اختيار أفضل العيادات من حيث الأداء فى محاولة الارتقاء بجودة التعامل والتقامل وتم ذلك باستخدام خمسة معايير : - الأنشطة التى نفذت للارتقاء بجودة الأداء - المعايير الشهرية باستخدام استمارة الإشراف

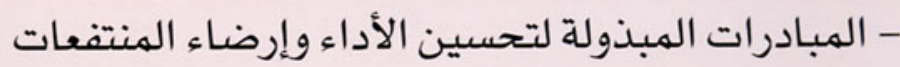
- استجابة مقدمى الخدمة لتتفيذ أهداف الدراسة

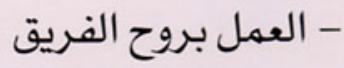

\section{- احتفالات التكريهم}

ولقد أجرى احتفال على مستوى كل محافظة بحضور مقدمى الخدمة بالعيادات المختارة للتكريم فى الإدارتين وفريق الإشراف على مستوى الإدارتين وفريق الإدارة ومديرى قطاع الأمومة والطفولة ومسئولى الإعلام وتم كذلك استضافة فرق الإشراف للمحافظة الأخرى فى حضور السيد وكيل وزارة الصحة والسكان بالمحافظة وقد تم تغطية أخبار الاحتفالات من خلال الإعلام المحلى . تم فى هذه الاحتفالات عرض للإنجازات من وجهة نظر الإدارات ثم مقدمى الخدمة وتم منح شهادات التقدير لمديرى العيادات المختارة ومقدمى الخدمة بها .

المرحلة الثالثة : مرحلة التدريب المستمر ومتابعة الأداء ( مايو - يوليو ا+.ب ) وقد تم فيها متابعة أداء مقدمى الخدمة بالعيادات مع التدريب المستمر وذلك من خلال زيارات الإشراف التيسيرى التى تمت بمعدلات أقل من المرحلة السابقة . 


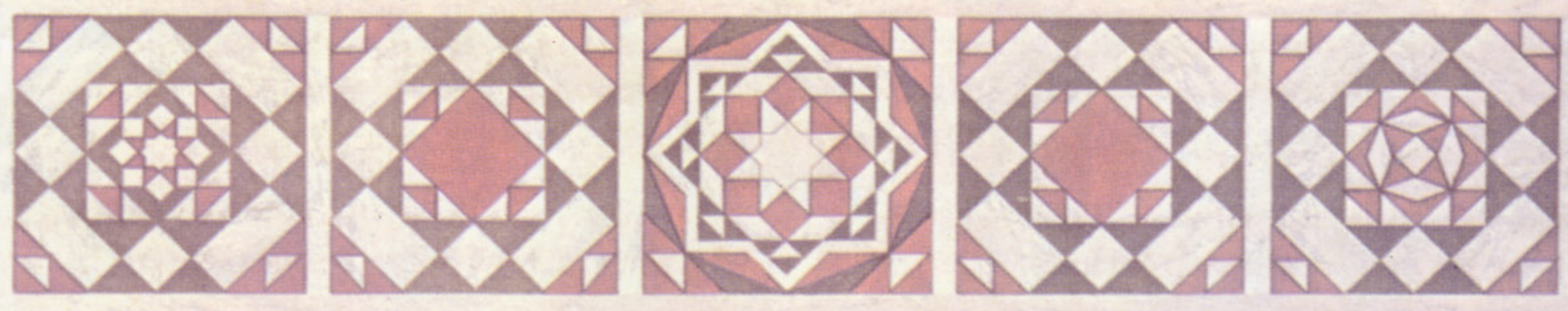

نجاحات الدراسة

كان لتدخلات الدراسة آثاراً إيجابية واسعة النطاق نوجزها فيما يلى : • ساعد التصميم المبتكر لتدخلات الدراسـة على استثارة الحماس وتتمية روح الفريق على كافة المستويات الذى انعكس على الابتكارات الفرديه والجماعيه وأتاح فرص استحداث حلول للمشاكل

$$
\text { والعراقيل }
$$

• استطاع فريق العمل نقل انطباعاته الإيجابية لمقدمى الخدمة على مستوى مراكز تتظيم الأسرة

مما حفز على استخدام الموارد المتاحة والاعتماد عليها وتطويعها لتحسين مستوى الخدمة . • أصبح التقييم الذاتى من أهم التدخلات التى أتاحت لكل فرد من أفراد مقدمى الخدمة تقييم التها

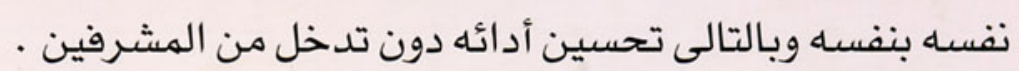

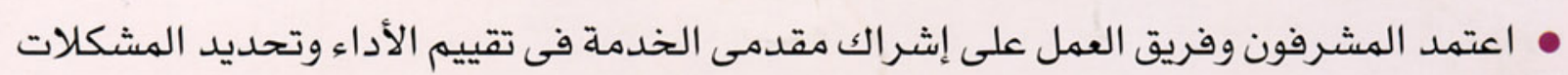
واقتراح الحلول وكذلك إشـراكهم فى وضع أهداف الارتقاء بكل مركز على حده مما أدى إلى خلق

$$
\text { جو من المشاركة الإيجابية مع الالتزام والإحساس بالملكيه والانتماء . }
$$

• ولقد أظهرت نتيجة الدراسـة بعد تتفيذ كافة التدخلات التحسن الإيجابى فى التعامل والتفاعل بين المنتفعات ومقدمى الخدمة وخصوصاً فى تحديد الاحتياجات الإنجابية للمنتفعة وتعريف الإبي المنتفعة الطريقة الصحيحة لاستخدام الوسيلة والآثار الجانبية المحتملة لها وكذلك المتابعة الصحيحة لاستخدام الوسيلة وكلها عوامل تؤثر على استمرارية استخدام الوسيلة .

ويـجدر الإشـارة إلى أن التعاون الوثيق بين فريق البحث ومججلس السكان الدولى ووزارة الصححة والسكان التى قدمت الدعم المعنوى والمؤسسى بإزالة المعوقات وحل المشكلات المات كان له عظيم الأثر فى نجاح الدراسة .

$$
\text { لمزيد من المعلومات رجاء الاتصال بـ : }
$$

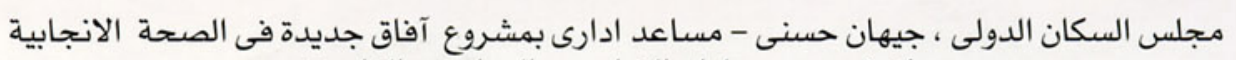

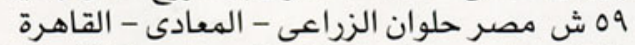

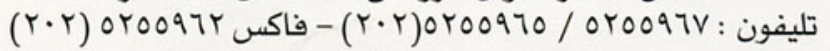
frontiers@pccairo.org : بريد الكترونى

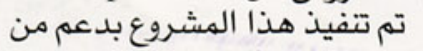
الوكالة الأمريكية للتتمية الدولية في ظل اتفاق التعاون رقفم: مشروع رقم: م800.13038 اتفاق التعاون

هذا و تعبر الاراء المذكورة بهذه المطبوعة من آراء المؤلفين فقط ولا تعكس رأى الهيئة الامريكية للتمية الدولية.

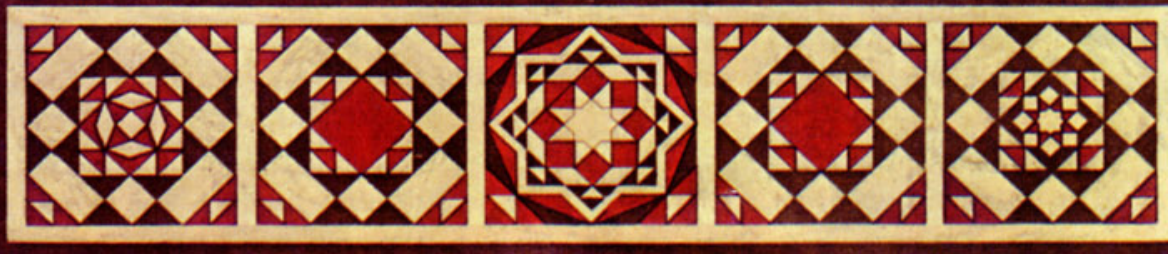

